\title{
On the Long-Range Dependence of Fractional Brownian Motion
}

\author{
Ming Li \\ School of Information Science \& Technology, East China Normal University, No. 500 Dong-Chuan Road, Shanghai 200241, China
}

Correspondence should be addressed to Ming Li; ming_lihk@yahoo.com

Received 5 May 2013; Accepted 15 May 2013

Academic Editor: Massimo Scalia

Copyright (C) 2013 Ming Li. This is an open access article distributed under the Creative Commons Attribution License, which permits unrestricted use, distribution, and reproduction in any medium, provided the original work is properly cited.

\begin{abstract}
This paper clarifies that the fractional Brownian motion, $B_{H}(t)$, is of long-range dependence (LRD) for the Hurst parameter $0<$ $H<1$ except $H=1 / 2$. In addition, we note that the fractional Brownian motion is positively correlated for $0<H<1$ except $H=1 / 2$. Moreover, we present a theorem to state that the differential or integral of a random function, $X(t)$, may substantially change the statistical dependence of $X(t)$. One example is that the differential of $B_{H}(t)$, in the domain of generalized functions, changes the LRD of $B_{H}(t)$ to be of short-range dependence (SRD) when $0<H<0.5$.
\end{abstract}

\section{Introduction}

Fractional Brownian motion ( $\mathrm{fBm})$ is widely used [1-10]. Its theory and applications attract the interests of researchers in various fields, ranging from telecommunications to biomedical engineering; see, for example, [11-44], simply citing a few.

There is a set of statistical properties of $\mathrm{fBm}$, such as nonstationarity and being nondifferentiable in the domain of ordinary functions [45]. Two properties, namely, nonstationarity and nondifferentiable property, are the basic properties of standard Brownian motion (Bm) [46-52], which is well known in the fields of time series as well as stochastic processes $[53,54]$. As the substantial generalization of $\mathrm{Bm}, \mathrm{fBm}$ has a property that $\mathrm{Bm}$ lacks, that is, its statistical dependence $[1-4,45]$. The measure of the statistical dependence of $\mathrm{fBm}$ is characterized by the Hurst parameter $H \in(0,1)$.

Note that the $\mathrm{fBm}$ for the Hurst parameter $H \in(0,1)$ and $H \neq 1 / 2$ is of LRD [11, 12, 45, 55, 56]. In addition, $\mathrm{fBm}$ is positively correlated for $H \in(0,1)$ but $H \neq 1 / 2$ [57]. However, the LRD property of $\mathrm{fBm}$ may be sometimes conservatively expressed. For example, the LRD property of $\mathrm{fBm}$ was restricted by $H \in(0.5,1)$ as can be seen from [58, page 2341] and [59, page 708]. For this reason, it may be meaningful to clarify, which this paper aims at.

The remaining paper is organized as follows. In Section 2, we describe that the range of $H$ for $\mathrm{fBm}$ to be of LRD is $H \in(0,1)$ and $H \neq 1 / 2$. Discussions are in Section 3 , which is followed by conclusions.

\section{FBm Is LRD for $0<H<1$ except $H=0.5$}

In what follows, a random function in general is denoted by $X(t)$ for $t \in(0, \infty)$. We denote $B_{H}(t)$ for $t \in(0, \infty)$ as $\mathrm{fBm}$ with $H \in(0,1)$.

Without generality losing, we assume that $X(t)$ is a random function with mean zero. The autocorrelation function $(\mathrm{ACF})$ of $X(t)$ is, for $t, s \in(0, \infty)$, denoted by

$$
C_{X X}(t, s)=E[X(t) X(s)] .
$$

By LRD $[1,2]$, we mean that

$$
\int_{0}^{\infty} C_{X X}(t, s) d t=\infty .
$$

If

$$
\int_{0}^{\infty} C_{X X}(t, s) d t<\infty,
$$

$X(t)$ is of short-range dependence (SRD).

Denote by $S_{X X}(\omega, t)$ the power spectrum density function (PSD) of $X(t)$. Denote by $F$ the operator of the Fourier transform. Then [60-64],

$$
S_{X X}(\omega, t)=F\left[C_{X X}(t, s)\right] .
$$

The LRD condition described in the frequency domain is expressed by

$$
\lim _{\omega \rightarrow 0} S_{X X}(\omega, s)=\infty .
$$


The above expression implies the property of $1 / f$ noise regarding random functions with LRD [1-4, 65-70]. On the other hand, $X(t)$ is of SRD if

$$
\lim _{\omega \rightarrow 0} S_{X X}(\omega, s)<\infty .
$$

Let $W^{-v}$ be the Weyl integral of order $v>0$. Then, for random function $X(t)$; see, for example, [71-75], one has

$$
W^{-v} X(t)=\frac{1}{\Gamma(v)} \int_{t}^{\infty}(u-t)^{v-1} X(u) d u .
$$

Thus, the fBm of the Weyl type is in the form:

$$
\begin{aligned}
B_{H}(t)-B_{H}(0)= & \frac{1}{\Gamma(H+1 / 2)} \\
& \times\left\{\int_{-\infty}^{0}\left[(t-u)^{H-0.5}-(-u)^{H-0.5}\right] d B(u)\right. \\
& \left.\quad+\int_{0}^{t}(t-u)^{H-0.5} d B(u)\right\} .
\end{aligned}
$$

Following [76], the PSD of the $\mathrm{fBm}$ of the Weyl type is expressed by

$$
S_{B_{H} B_{H}}(\omega, t)=\frac{1}{|\omega|^{2 H+1}}\left(1-2^{1-2 H} \cos 2 \omega t\right) .
$$

Therefore, we have the following theorem.

Theorem 1. FBm is of $L R D$ for $H \in(0,1)$ except $H=1 / 2$.

Proof. Because $\lim _{\omega \rightarrow 0} S_{B_{H} B_{H}}(\omega, t)=\infty$ for all $t>0$ and for $H \in(0,1)$ except $H=1 / 2$, the theorem holds.

As a matter of fact, $\mathrm{fBm}$ reduces to the standard $\mathrm{Bm}$ if $H=1 / 2$. The PSD of BM, see [11], is given by

$$
S_{B_{1 / 2} B_{1 / 2}}(t, \omega)=\frac{1}{\omega^{2}}(1-\cos 2 \omega t) .
$$

Thus,

$$
\lim _{\omega \rightarrow 0} S_{B_{1 / 2} B_{1 / 2}}(t, \omega)=2 t^{2} \neq \infty
$$

From the theorem, we have the following corollary.

Corollary 2. FBm is not SRD for $H \in(0,1)$.

In passing, we mention that the ACF of $B_{H}(t)$ of the Weyl type is in the form:

$$
\begin{aligned}
C_{B_{H} B_{H}}(t, s)= & \frac{V_{H}}{(H+1 / 2) \Gamma(H+1 / 2)} \\
& \times\left[|t|^{2 H}+|s|^{2 H}-|t-s|^{2 H}\right],
\end{aligned}
$$

where $V_{H}$ is the strength of $B_{H}(t)$. It is given by

$$
V_{H}=\operatorname{Var}\left[B_{H}(1)\right]=\Gamma(1-2 H) \frac{\cos \pi H}{\pi H} .
$$

Following [57, page 4], we have the following remark.

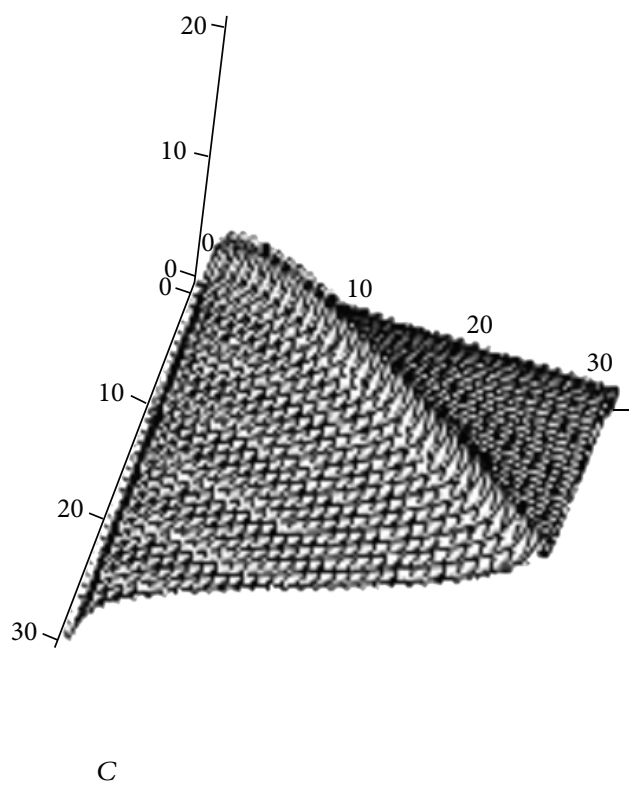

Figure 1: ACF of fBm for $t, s=0,1, \ldots, 30$ and $H=0.2$.

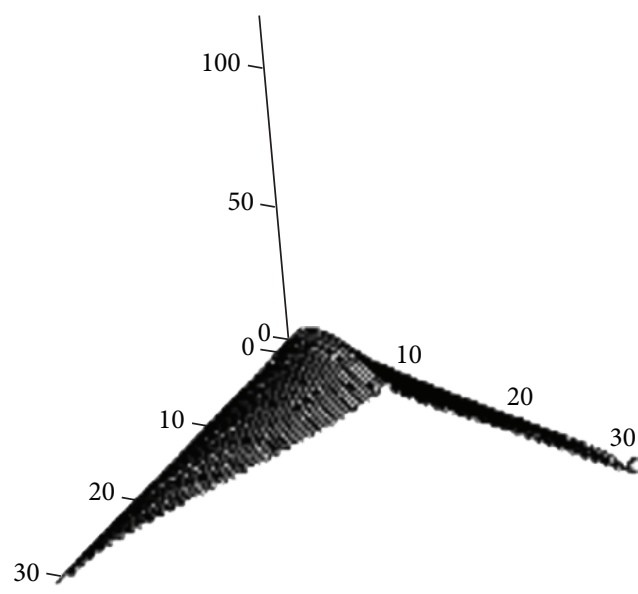

C

Figure 2: ACF of fBm for $t, s=0,1, \ldots, 30$ and $H=0.4$.

Remark 3. The ACF of $\mathrm{fBm}$ is positively correlated for $H \in$ $(0,1)$ except $H=1 / 2$. That is, $C_{B_{H} B_{H}}(t, s) \geq 0$ for $t, s \in$ $(0, \infty)$. Figures 1 and 2 indicate the plots of $C_{B_{H} B_{H}}(t, s)$ for $t, s=0,1, \ldots, 30$ with $H=0.2$ and 0.4 , respectively.

\section{Discussions}

Let $G_{H}(t)$ be the fractional Gaussian noise (fGn). Then, in the domain of generalized functions over the Schwartz space of test functions [45], we write

$$
G_{H}(t)=\frac{d B_{H}(t)}{d t}
$$


Denote by $C_{G_{H} G_{H}}(\tau, s)$ the ACF of $G_{H}(t)$. Then, for $\varepsilon>0[19$, 45], one has

$$
\begin{aligned}
C_{G_{H} G_{H}}(\tau ; \varepsilon)= & \frac{V_{H} \varepsilon^{2 H-2}}{2} \\
& \times\left[\left(\frac{|\tau|}{\varepsilon}+1\right)^{2 H}+\left|\frac{|\tau|}{\varepsilon}-1\right|^{2 H}-2\left|\frac{\tau}{\varepsilon}\right|^{2 H}\right]
\end{aligned}
$$

From the contents in Section 2, we have the following theorem.

Theorem 4. Let $X(t)$ be a random function. Then, the statistical dependence of $d X(t) / d t$ may substantially differ from that of $X(t)$, where the differential is in the domain of generalized functions.

Proof. To prove the theorem, we only need an example to show it. Let $X(t)=B_{H}(t)$. Then, $d X(t) / d t=G_{H}(t)$. It is well known that fGn is LRD when $H \in(0.5,1)$ as $C_{G_{H} G_{H}}$ is nonintegrable if $H \in(0.5,1)$. On the other hand, for $H \in$ $(0,0.5)$, the integral of $C_{G_{H} G_{H}}$ is zero. Hence, fGn is SRD when $H \in(0,0.5)$. In passing, we note that $C_{G_{H} G_{H}}(\tau ; \varepsilon)$ changes its sign and becomes negative for some $\tau$ proportional to $\varepsilon$ in this parameter domain [45, page 434]. Since $B_{H}(t)$ is LRD for $H \in(0,1)$ except $H=1 / 2$, the statistical dependence of $G_{H}(t)$ substantially differs from that of $B_{H}(t)$. This completes the proof. below.

From Theorem 4, we immediately obtain the corollary

Corollary 5. Let $X(t)$ be a random function. Then, the statistical dependence of $D^{-1} X(t)$ may substantially differ from that of $X(t)$, where $D^{-1}$ is the integral operator of order one.

Proof. Let $X(t)=G_{H}(t)$. Then, $D^{-1} X(t)=B_{H}(t)$. Since $B_{H}(t)$ is LRD for $H \in(0,0.5)$ while $G_{H}(t)$ is SRD when $H \in$ $(0,0.5)$, one sees that the statistical dependence of $D^{-1} X(t)$ substantially differs from that of $X(t)$. Thus, Corollary 5 results.

\section{Conclusions}

We have clarified that $\mathrm{fBm}$ is LRD and positively correlated for $H \in(0,1)$ except $H=1 / 2$. In addition, we have proved that the differential or integral of a random function may considerably change its statistical dependence.

\section{Acknowledgments}

This work was supported in part by the 973 plan under the project Grant no. 2011CB302800 and by the National Natural Science Foundation of China under the project Grants nos. 61272402, 61070214, and 60873264 .

\section{References}

[1] B. B. Mandelbrot, Gaussian Self-Affinity and Fractals, Springer, New York, NY, USA, 2001.

[2] J. Beran, Statistics for Long-Memory Processes, Chapman and Hall, New York, NY, USA, 1994.

[3] J. Levy-Vehel, E. Lutton, and C. Tricot, Eds., Fractals in Engineering, Springer, 1997.

[4] P. Doukhan, G. Oppenheim, and M. S. Taqqu, Eds., Theory and Applications of Long-Range Dependence, Birkhäuser, Boston, Mass, USA, 2002.

[5] J. B. Bassingthwaighte, L. S. Liebovitch, and B. J. West, Fractal Physiology, Oxford University Press, 1994.

[6] J.-P. Bouchaud and A. Georges, "Anomalous diffusion in disordered media: statistical mechanisms, models and physical applications," Physics Reports, vol. 195, no. 4-5, pp. 127-293, 1990.

[7] W. Willinger and V. Paxson, "Where mathematics meets the internet," Notices of the American Mathematical Society, vol. 45, no. 8, pp. 961-970, 1998.

[8] V. Paxson and S. Floyd, "Wide area traffic: the failure of Poisson modeling," IEEE/ACM Transactions on Networking, vol. 3, no. 3, pp. 226-244, 1995

[9] S. C. Lim and L. P. Teo, "Modeling single-file diffusion with step fractional Brownian motion and a generalized fractional Langevin equation," Journal of Statistical Mechanics, vol. 2009, no. 8, Article ID P08015, 24 pages, 2009.

[10] S. Kim, S. Y. Nam, and D. K. Sung, "Effective bandwidth for a single server queueing system with fractional Brownian input," Performance Evaluation, vol. 61, no. 2-3, pp. 203-223, 2005.

[11] V. M. Sithi and S. C. Lim, "On the spectra of Riemann-Liouville fractional Brownian motion," Journal of Physics, vol. 28, no. 11, pp. 2995-3003, 1995.

[12] S. V. Muniandy and S. C. Lim, "Modeling of locally self-similar processes using multifractional Brownian motion of RiemannLiouville type," Physical Review E, vol. 63, no. 4, part 2, Article ID 461047, 2001.

[13] D. Feyel and A. de La Pradelle, "On fractional Brownian processes," Potential Analysis, vol. 10, no. 3, pp. 273-288, 1999.

[14] R. F. Peltier and J. Levy-Vehel, "Multifractional Brownian motion: definition and preliminaries results," INRIA TR 2645, 1995.

[15] T. G. Sinai, "Distribution of the maximum of a fractional Brownian motion," Russian Mathematical Surveys, vol. 52, no. 2, pp. 119-138, 1997.

[16] S. C. Lim and S. V. Muniandy, "On some possible generalizations of fractional Brownian motion," Physics Letters A, vol. 266, no. 2-3, pp. 140-145, 2000.

[17] M. D. Ortigueira and A. G. Batista, "On the relation between the fractional Brownian motion and the fractional derivatives," Physics Letters A, vol. 372, no. 7, pp. 958-968, 2008.

[18] S. C. Lim and L. P. Teo, "Weyl and Riemann-Liouville multifractional Ornstein-Uhlenbeck processes," Journal of Physics A, vol. 40, no. 23, pp. 6035-6060, 2007.

[19] M. Li and S. C. Lim, "A rigorous derivation of power spectrum of fractional Gaussian noise," Fluctuation and Noise Letters, vol. 6, no. 4, pp. C33-C36, 2006.

[20] M. Li and W. Zhao, "On bandlimitedness and lag-limitedness of fractional Gaussian noise,” Physica A, vol. 392, no. 9, pp. 19551961, 2013.

[21] M. Li and W. Zhao, "Quantitatively investigating locally weak stationarity of modified multifractional Gaussian noise," Physica A, vol. 391, no. 24, pp. 6268-6278, 2012. 
[22] V. Paxson, "Fast approximate synthesis of fractional gaussian noise for generating self-similar network traffic," ACM SIGCOMM Computer Communication Review, vol. 27, no. 5, pp. 518, 1997.

[23] C. Cattani, M. Scalia, E. Laserra, I. Bochicchio, and K. K. Nandi, "Correct light deflection in Weyl conformal gravity," Physical Review D, vol. 87, no. 4, Article ID 47503, 4 pages, 2013.

[24] C. Cattani, "Harmonic wavelet approximation of random, fractal and high frequency signals," Telecommunication Systems, vol. 43, no. 3-4, pp. 207-217, 2010.

[25] C. Cattani, G. Pierro, and G. Altieri, "Entropy and multifractality for the myeloma multiple TET 2 gene," Mathematical Problems in Engineering, vol. 2012, Article ID 193761, 14 pages, 2012.

[26] C. Cattani, "Fractals and hidden symmetries in DNA," Mathematical Problems in Engineering, vol. 2010, Article ID 507056, 31 pages, 2010.

[27] S. Hu, Z. Liao, and W. Chen, "Sinogram restoration for lowdosed X-ray computed tomography using fractional-order Perona-Malik diffusion," Mathematical Problems in Engineering, vol. 2012, Article ID 391050, 13 pages, 2012.

[28] Z. W. Liao, S. X. Hu, D. Sun, and W. F. Chen, "Enclosed Laplacian operator of nonlinear anisotropic diffusion to preserve singularities and delete isolated points in image smoothing," Mathematical Problems in Engineering, vol. 2011, Article ID 749456, 15 pages, 2011.

[29] J. W. Yang, Y. J. Chen, and M. Scalia, "Construction of affine invariant functions in spatial domain," Mathematical Problems in Engineering, vol. 2012, Article ID 690262, 11 pages, 2012.

[30] H. E. Stanley, S. V. Buldyrev, A. L. Goldberger, S. Havlin, C.-K. Peng, and M. Simons, "Long-range power-law correlations in condensed matter physics and biophysics," Physica A, vol. 200, no. 1-4, pp. 4-24, 1993.

[31] B. Podobnik, P. C. Ivanov, K. Biljakovic, D. Horvatic, H. E. Stanley, and I. Grosse, "Fractionally integrated process with powerlaw correlations in variables and magnitudes," Physical Review E, vol. 72, no. 2, Article ID 026121, 7 pages, 2005.

[32] A. Carbone, G. Castelli, and H. E. Stanley, “Time-dependent Hurst exponent in financial time series," Physica A, vol. 344, no. 1-2, pp. 267-271, 2004.

[33] Z. Chen, P. C. Ivanov, K. Hu, and H. E. Stanley, "Effect of nonstationarities on detrended fluctuation analysis," Physical Review E, vol. 65, no. 4, Article ID 041107, 15 pages, 2002.

[34] C. Toma, "Advanced signal processing and command synthesis for memory-limited complex systems," Mathematical Problems in Engineering, vol. 2012, Article ID 927821, 13 pages, 2012.

[35] E. G. Bakhoum and C. Toma, "Specific mathematical aspects of dynamics generated by coherence functions," Mathematical Problems in Engineering, vol. 2011, Article ID 436198, 10 pages, 2011.

[36] E. G. Bakhoum and C. Toma, "Mathematical transform of traveling-wave equations and phase aspects of quantum interaction," Mathematical Problems in Engineering, Article ID 695208, 15 pages, 2010.

[37] G. Korvin, Fractal Models in the Earth Science, Elsevier, 1992.

[38] E. E. Peters, Fractal Market Analysis-Applying Chaos Theory to Investment and Economics, John Wiley \& Sons, 1994.

[39] S.-C. Liu and S. Chang, "Dimension estimation of discrete-time fractional Brownian motion with applications to image texture classification," IEEE Transactions on Image Processing, vol. 6, no. 8, pp. 1176-1184, 1997.
[40] S. Chang, S.-T. Mao, S.-J. Hu, W.-C. Lin, and C.-L. Cheng, "Studies of detrusor-sphincter synergia and dyssynergia during micturition in rats via fractional brownian motion," IEEE Transactions on Biomedical Engineering, vol. 47, no. 8, pp. 10661073, 2000.

[41] C. Fortin, R. Kumaresan, W. Ohley, and S. Hoefer, "Fractal dimension in the analysis of medical images," IEEE Engineering in Medicine and Biology Magazine, vol. 11, no. 2, pp. 65-71, 1992.

[42] A. Eke, E. P. Herman, J. B. Bassingthwaighte et al., "Physiological time series: distinguishing fractal analysis noises from motions," European Journal of Physiology, vol. 439, no. 4, pp. 403-415, 2000.

[43] G. M. Raymond, D. B. Percival, and J. B. Bassingthwaighte, "The spectra and periodograms of anti-correlated discrete fractional Gaussian noise," Physica A, vol. 322, pp. 169-179, 2003.

[44] H. E. Schepers, J. H. G. M. van Beek, and J. B. Bassingthwaighte, "Four methods to estimate the fractal dimension from selfaffine signals," IEEE Engineering in Medicine and Biology Magazine, vol. 11, no. 2, pp. 57-64, 1992.

[45] B. B. Mandelbrot and J. W. Van Ness, "Fractional Brownian motions, fractional noises and applications," SIAM Review, vol. 10, pp. 422-437, 1968.

[46] G. E. Uhlenbeck and L. S. Ornstein, "On the theory of the Brownian motion," Physical Review, vol. 36, no. 5, pp. 823-841, 1930.

[47] T. Hida, Brownian Motion, Springer, 1980.

[48] J. Dunkel and P. Hänggi, "Relativistic Brownian motion,” Physics Reports, vol. 471, no. 1, pp. 1-73, 2009.

[49] M. Peligrad and S. Utev, "Another approach to Brownian motion," Stochastic Processes and their Applications, vol. 116, no. 2, pp. 279-292, 2006.

[50] E. Frey and K. Kroy, "Brownian motion: a paradigm of soft matter and biological physics," Annalen der Physik, vol. 14, no. 1-3, pp. 20-50, 2005.

[51] P. Hänggi, F. Marchesoni, and F. Nori, "Brownian motors," Annalen der Physik, vol. 14, no. 1-3, pp. 51-70, 2005.

[52] F. Cecconi, M. Cencini, M. Falcioni, and A. Vulpiani, "Brownian motion and diffusion: from stochastic processes to chaos and beyond," Chaos, vol. 15, no. 2, Article ID 026102, p. 9, 2005.

[53] A. Papoulis, Probability, Random Variables and Stochastic Processes, McGraw-Hill Book, 2nd edition, 1984.

[54] W. A. Fuller, Introduction to Statistical Time Series, John Wiley \& Sons, New York, NY, USA, 2nd edition, 1996.

[55] S. C. Lim and S. V. Muniandy, "Generalized Ornstein-Uhlenbeck processes and associated self-similar processes," Journal of Physics A, vol. 36, no. 14, pp. 3961-3982, 2003.

[56] H. Qian, "Fractional brownian motion and fractional gaussian noise," Lecture Notes in Physics, vol. 621, pp. 22-33, 2003.

[57] F. Aurzada and C. Baumgarten, "Persistence of fractional Brownian motion with moving boundaries and applications," Journal of Physics A, vol. 46, no. 12, Article ID 125007, 2013.

[58] V. Maroulas and J. Xiong, "Large deviations for optimal filtering with fractional Brownian motion," Stochastic Processes and their Applications, vol. 123, no. 6, pp. 2340-2352, 2013.

[59] R. Scheffer and R. Maciel Filho, "The fractional Brownian motion as a model for an industrial airlift reactor," Chemical Engineering Science, vol. 56, no. 2, pp. 707-711, 2001.

[60] M. Sun, C. C. Li, L. N. Sekhar, and R. J. Sclabassi, "Wigner spectral analyzer for nonstationary signals," IEEE Transactions on Instrumentation and Measurement, vol. 38, no. 5, pp. 961966, 1989. 
[61] M. B. Priestley, "Evolutionary spectra and non-stationary processes," Journal of the Royal Statistical Society B, vol. 27, pp. 204237, 1965.

[62] J. S. Bendat and A. G. Piersol, Random Data: Analysis and Measurement Procedure, John Wiley \& Sons, 3rd edition, 2010.

[63] J. Xiao and P. Flandrin, "Multitaper time-frequency reassignment for nonstationary spectrum estimation and chirp enhancement," IEEE Transactions on Signal Processing, vol. 55, no. 6, pp. 2851-2860, 2007.

[64] P. Borgnat, P. Flandrin, P. Honeine, C. Richard, and J. Xiao, "Testing stationarity with surrogates: a time-frequency approach," IEEE Transactions on Signal Processing, vol. 58, no. 7, pp. 3459-3470, 2010.

[65] M. Li and W. Zhao, "On $1 / f$ noise," Mathematical Problems in Engineering, vol. 2012, Article ID 673648, 22 pages, 2012.

[66] K. Fraedrich, U. Luksch, and R. Blender, " $1 / f$ model for long-time memory of the ocean surface temperature," Physical Review E, vol. 70, no. 3, Article ID 037301, pp. 1-4, 2004.

[67] W. T. Li and D. Holste, "Universal $1 / f$ noise, crossovers of scaling exponents, and chromosome-specific patterns of guaninecytosine content in DNA sequences of the human genome," Physical Review E, vol. 71, no. 4, Article ID 041910, 9 pages, 2005.

[68] F. N. Hooge, “1/ $f$ noise," Physica B, vol. 83, no. 1, pp. 14-23, 1976.

[69] M. S. Keshner, " $1 / f$ noise," Proceedings of the IEEE, vol. 70, no. 3, pp. 212-218, 1982.

[70] G. Aquino, M. Bologna, P. Grigolini, and B. J. West, "Beyond the death of linear response: $1 / f$ optimal information transport," Physical Review Letters, vol. 105, no. 6, Article ID 069901, 1 page, 2010.

[71] J. Klafter, S. C. Lim, and R. Metzler, Fractional Dynamics: Recent Advances, World Scientific, 2012.

[72] A. A. Kilbas, H. M. Srivastava, and J. J. Trujillo, Theory and Applications of Fractional Differential Equations, Elsevier Science, Amsterdam, The Netherlands, 2006.

[73] S. Samko, A. A. Kilbas, and D. I. Maritchev, Integrals and Derivatives of the Fractional Order and Some of Their Applications, Gordon and Breach, Amsterdam, The Netherlands, 1993.

[74] G. M. Zaslavsky, Hamiltonian Chaos and Fractional Dynamics, Oxford University Press, Oxford, UK, 2008.

[75] R. C. Blei, Analysis in Integer and Fractional Dimensions, University Press, Cambridge, UK, 2003.

[76] P. Flandrin, "On the spectrum of fractional Brownian motions," IEEE Transactions on Information Theory, vol. 35, no. 1, pp. 197199, 1989. 


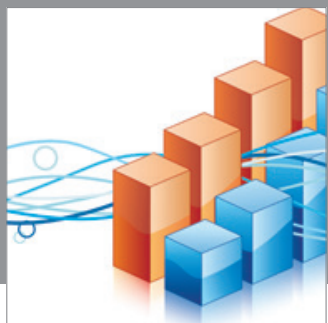

Advances in

Operations Research

mansans

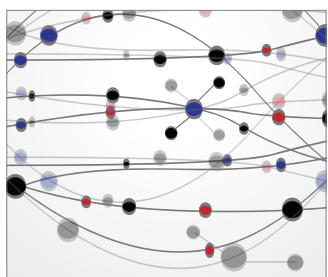

The Scientific World Journal
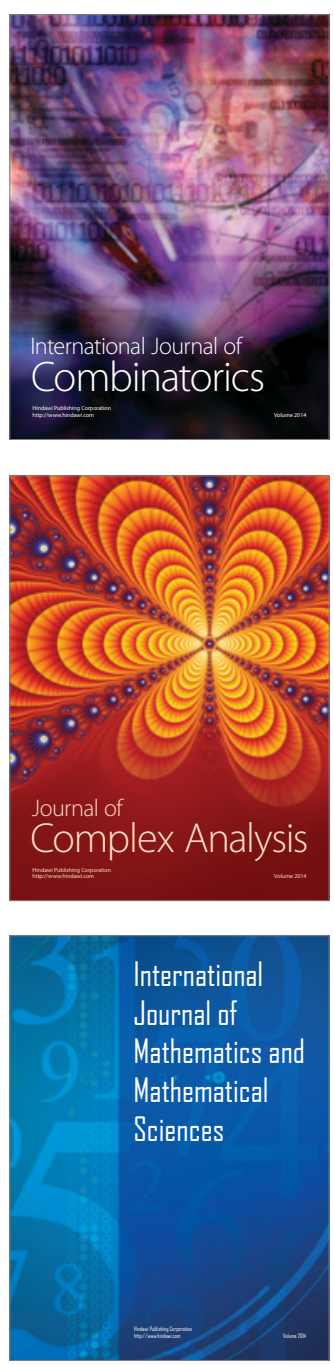
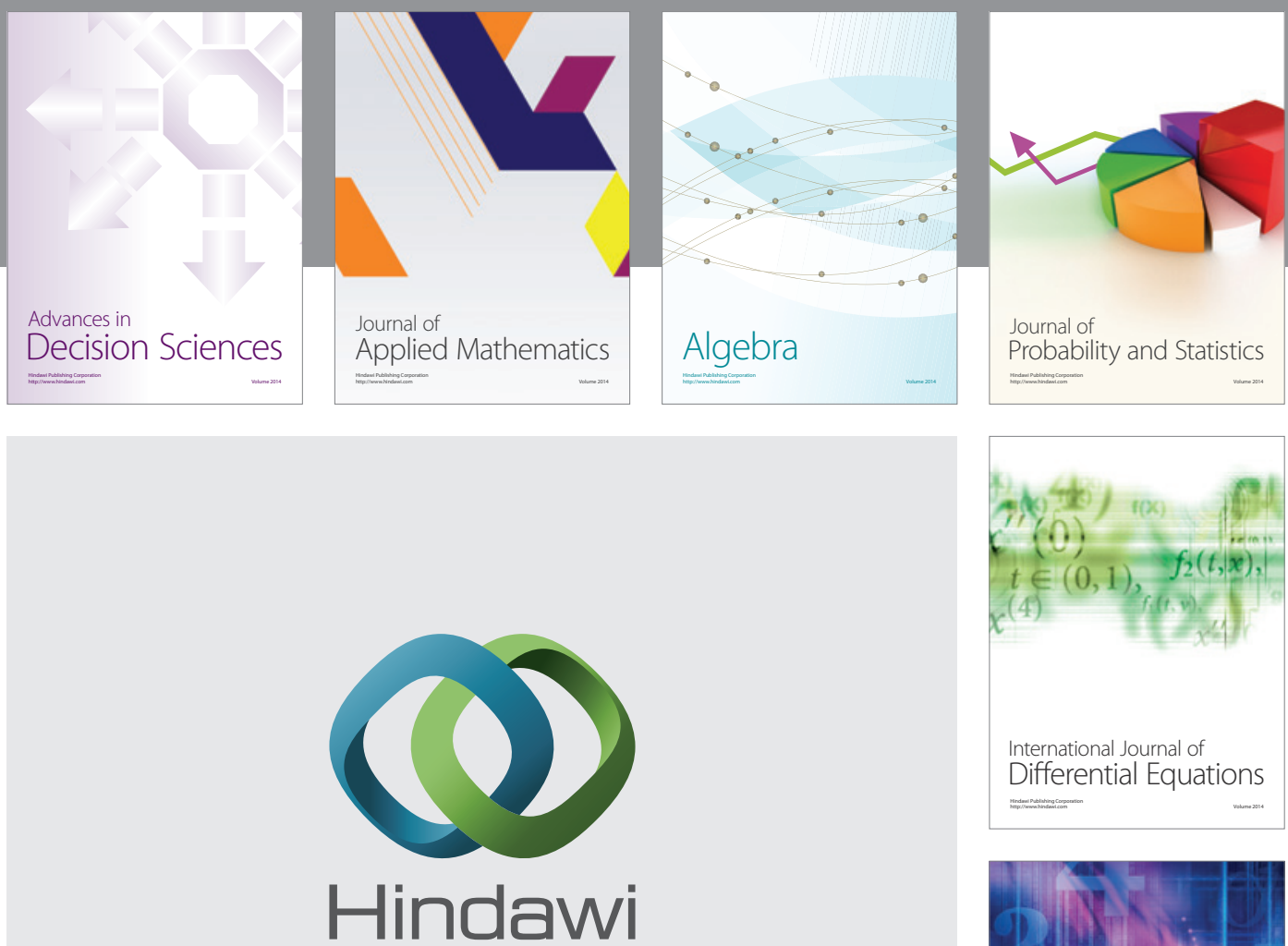

Submit your manuscripts at http://www.hindawi.com
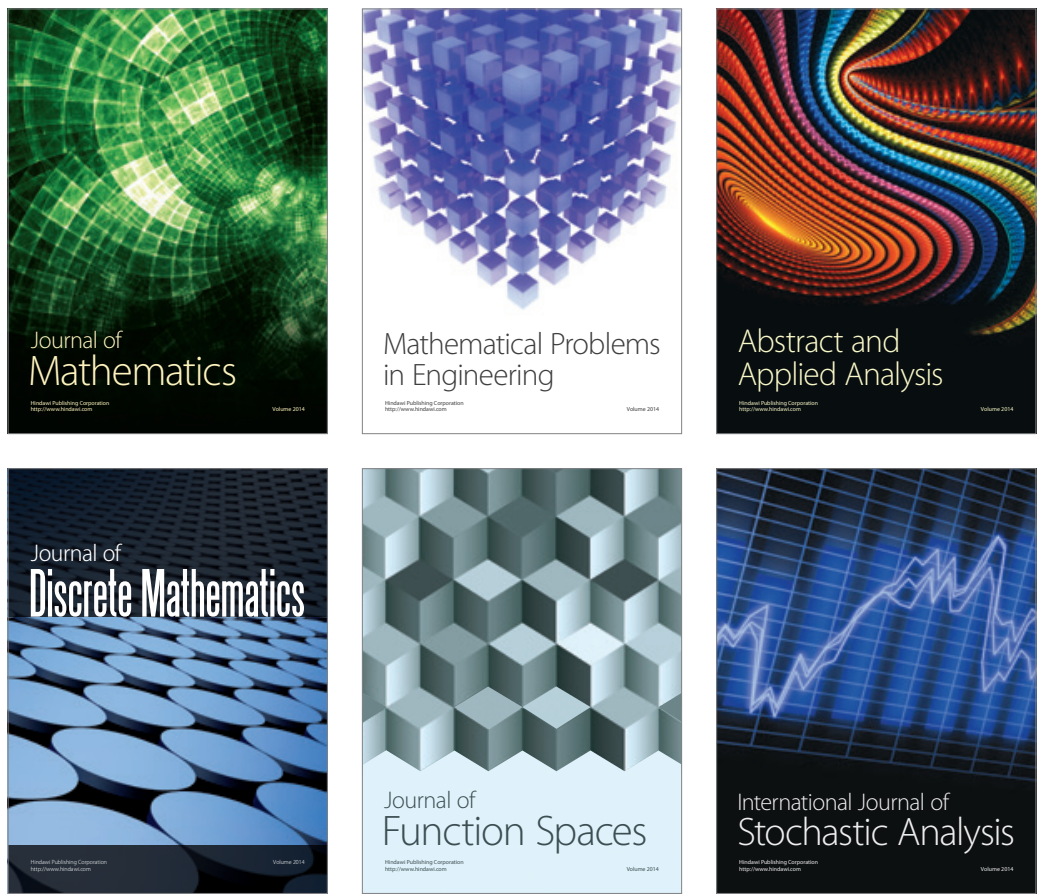

Journal of

Function Spaces

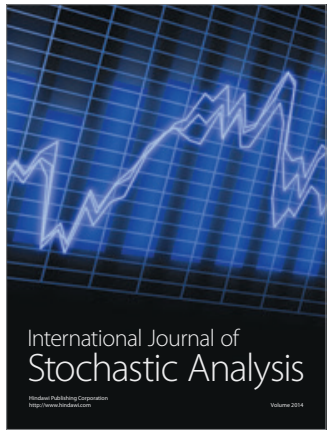

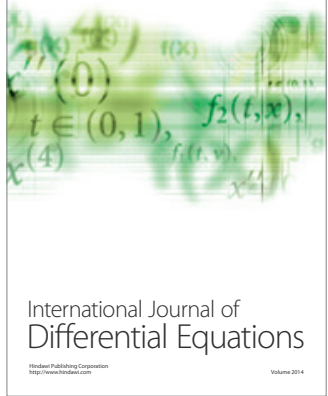
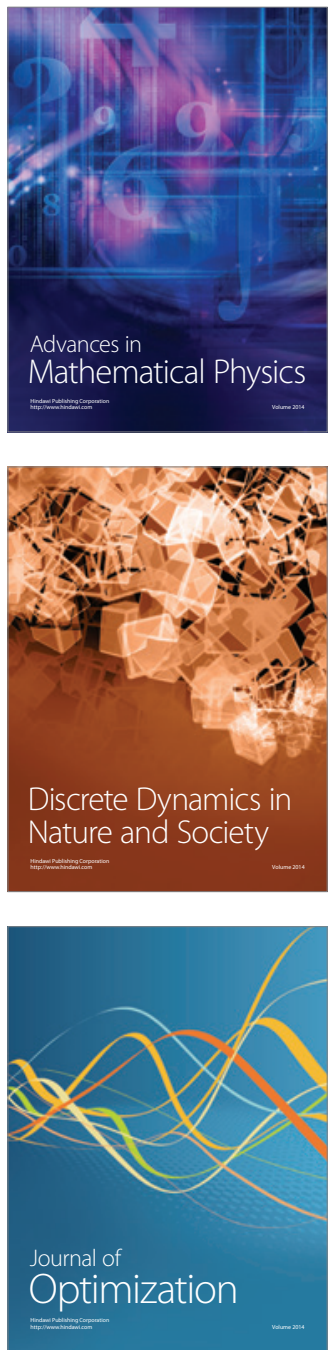